\title{
New approach using an loT robot to oversight the smart home environment
}

\section{Nouvelle approche utilisant un robot loT pour la surveillance de l'environnement d'une maison intelligente}

\author{
Meftah Zouai', Okba Kazar ${ }^{1}$, Belgacem Haba², Guadalupe Ortiz ${ }^{3}$, Nadia Kabachi ${ }^{4}$ \\ ${ }^{1}$ University of Mohamed Khider, Laboratory LINFI, Biskra, Algeria, meftah.zouai@univ-biskra.dz, o.kazar@univ-biskra.dz \\ ${ }^{2}$ Invensas Corp., San Jose, CA, USA, guadalupe.ortiz@uca.es \\ ${ }^{3}$ University of Cadiz, Laboratory UCASE, Cadiz, Spain, bhaba@invensas.com \\ ${ }^{4}$ University of Lyon 1, Laboratory ERIC, Lyon, France, nadia.kabachi@univ-lyon1.fr
}

\begin{abstract}
Smart environment needs a lot of sensors in different places. The cost of these sensors is expensive, and to reduce this cost we proposed a mobile robot. This robot carries a range of different sensors that will sense the surrounding environment and send data to the objects in the environment. Since our robot consist of a set of sensors, this can eliminate the possibility of using a huge number of sensor and placing those senssors on all the environment. The process of communication between the robot and the objects in the environment through the MQTT protocol. The IOT Things can control the robot directly through orders received by the robot.

RÉSUMÉ. L'environnement intelligent a besoin de beaucoup de capteurs à différents endroits. Le prix de ces capteurs est coûteux et, pour réduire ce coût, nous avons proposé un robot mobile. Ce robot porte une gamme de capteurs différents qui vont détecter l'environnement global et envoyer des données aux objets de l'environnement. Étant donné que notre robot se compose d'un ensemble de capteurs, cela peut éliminer la possibilité d'utiliser un grand nombre de capteurs et de les placer sur tout l'environnement. Le processus de communication entre le robot et les objets de l'environnement se déroule via le protocole MQTT. Les objets de L'loT peuvent contrôler le robot directement via les commandes reçues par le robot.

KEYWORDS. Internet of things, IOT Robot, Sensing, Smart home.

MOTS-CLÉS. Internet des objets, robot loT, surveillance, maison intelligente.
\end{abstract}

\section{Introduction}

Internet of things [ALJ 17, SAL 18a] is a network of objects mainly supported by electronic devices and electronic components such as sensors and electronic cards. These objects can be physical and virtual devices, sensors or actuators. Smart environment needs a lot of sensors and in different places. The cost of these sensors is expensive. To reduce the cost of these sensors, we proposed a mobile robot that carries a range of different sensors that will sense the surrounding environment and send information and data to the objects in the environment [VER 17, SAL 18b] Using this robot, we do not need sensors everywhere, but sensors are moving everywhere in the environment. The process of communication between the robot and the IoT objects in the environment through the MQTT protocol [NAI 17]. The IoT Things can control the robot directly through orders received by the robot.

We have implemented this Robot with a Raspberry Pi B perfected electronic card which has both sensors and Arduino UNO card for robot movement management [WAR 11]. It is also equipped with sensors to protect the robot from colliding with barriers and obstructions. These sensors also help the robot to overcome the impediments autonomously and help to draw a partial outline of the surrounding environment where the scheme is a circle of diameter of 4 meters, and the robot control carried out via a smartphone. The commands sent via the local network to robot if the smartphone and the robot are connected in the same Gateway. If the robot in the remote site, he will receives the commands via public network Internet [ALS XX ]. 
We provided the robot with a steerable camera to view the environment. It is controlled remotely by sent commands. The camera server (motion) sends the captured images to the user in real time and directly. Motion is a fairly complete surveillance system. It is extremely customizable: motion detection, frame-by-frame recording, video recording, time lapse.

The robot has a three main parties: displacement module; this is the module that has the role of managing the robot move [PIY 13], communication module: this module is for the exchange of information and commands between the robot and the user, sensing module; this module has a role of the link between the real world (the environment) and the digital world (the information world) by the transfer of real information (physical) to the digital information that can be treated and stored [MER $16]$.

\section{Proposed Architecture}

Robot control via smart phone[BOZ 15]. Orders sent via the smart phone to the local network robot. If the smart phone and the robot connected in the same model (WIFI router). If the robot in a remote site the robot receives the commands via internet Public network.

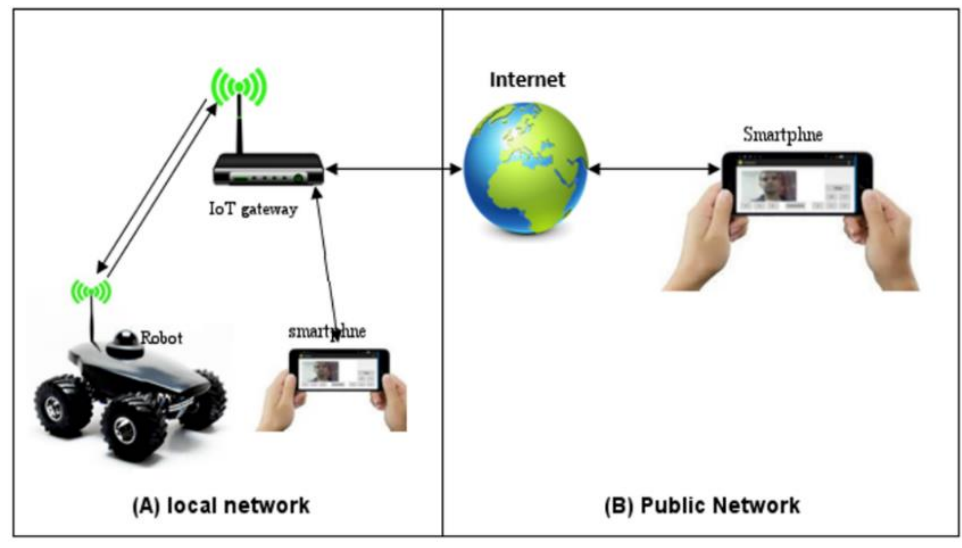

Fig. 1. Proposed Architecture

\section{Global functioning proposed architecture}

In our global functioning architecture proposed, there is three main parties, each part contains components and functions different from the other parts.

Our robot is made up of three vital parts:

The displacement module: is the module responsible for the movement of the robot and moving it from place to place. This module is turning the electric energy into kinetic energy through the motors in the robot.

The sensing module: is the module responsible for sensing the external medium. This unit converts the physical values of the environment into numerical values [CHE 70] that are capable of storage and analysis. This module connects the real world to the digital world.

The communication module is the module responsible for robot interaction with the user by receiving orders, reporting and images about the environment. 


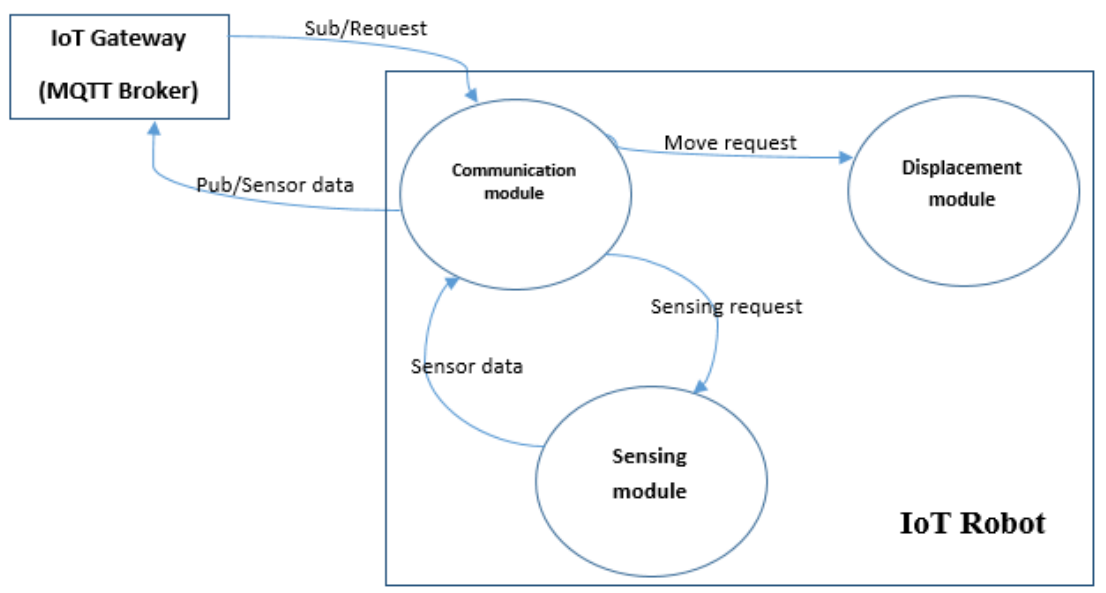

Fig 2. The loT robot modules

\subsection{Status diagram}

This diagram shows how the robot parts interact with each other to perform their goals ideally

- The communication module receives orders from the user These things are to be the initial status.

- The communication module will direct the order according to its type. If it is an order for moving or sensing command.

- If it is a matter of movement, the displacement module will execute it directly.

- The sensing module senses the environment through the sensors and takes pictures of the environment and send them directly and in real time to the user.

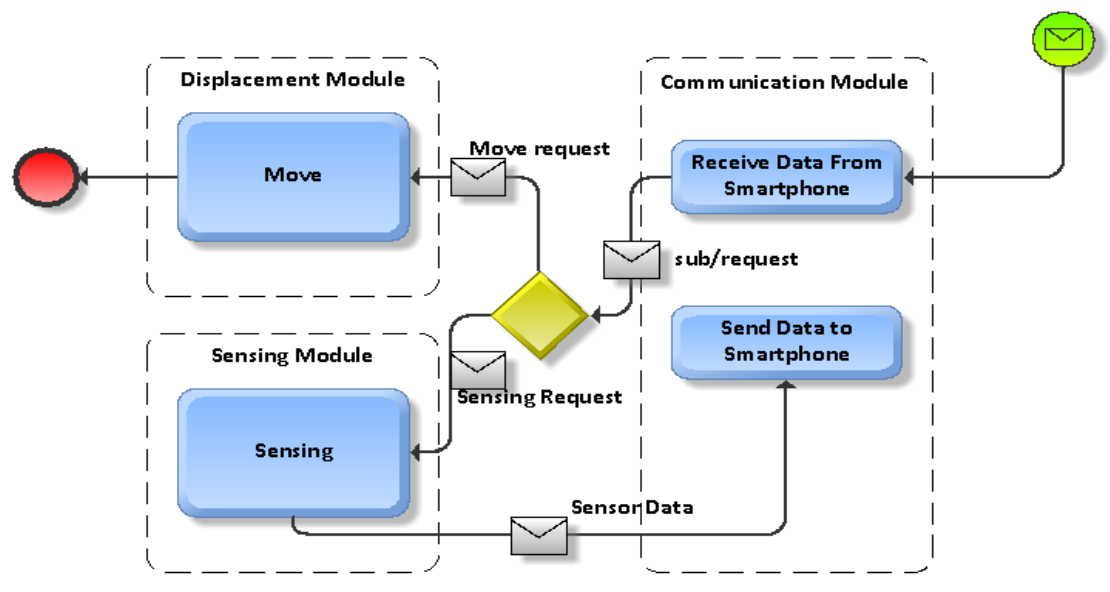

Fig 3. The loT robot modules

\section{Implementation}

In this section we present the most important electronic parts and software in the robot. The electronic Robot circuit

We used a motor to control the robot wheels (front and rear). We made the complete wiring of our hardware with Arduino and Raspberry Pi [ZHA 15 ][MAK 14] by attaching them with a serial cable for the transfer of information.

Raspberry Pi and Arduino communication: The commands received by the WiFi sensor on the Raspberry Pi using the MQTT protocol the Raspberry Pi make a redirection of commands to the Arduino via the serial port (RX, TX) [YAN 15]. 
Wiring the L293D with DC motor : L293D tabs 2, 7, 8 are connected to pins 2,9 and 10 of the Arduino, and the DC motor with tabs 3,6 to control the motor direction (front and rear).

Wiring of servomotors: Servo drive [11] pin is connected with Arduino pin 8 which is responsible for camera rotation, and also the second servo drive pin with Arduino pin 7 which is responsible for the orientation of the wheels.

Complete wiring with the Arduino: We do the complete wiring with the Arduino, we connect the anode of the red LEDs with the pin 4 and their cathode with (GND) .and also the anode of the white LEDs with the pin 3 and their cathode with (GND). We can control the power on and off of the white LEDs, but the red LEDs come on when the robot stops.

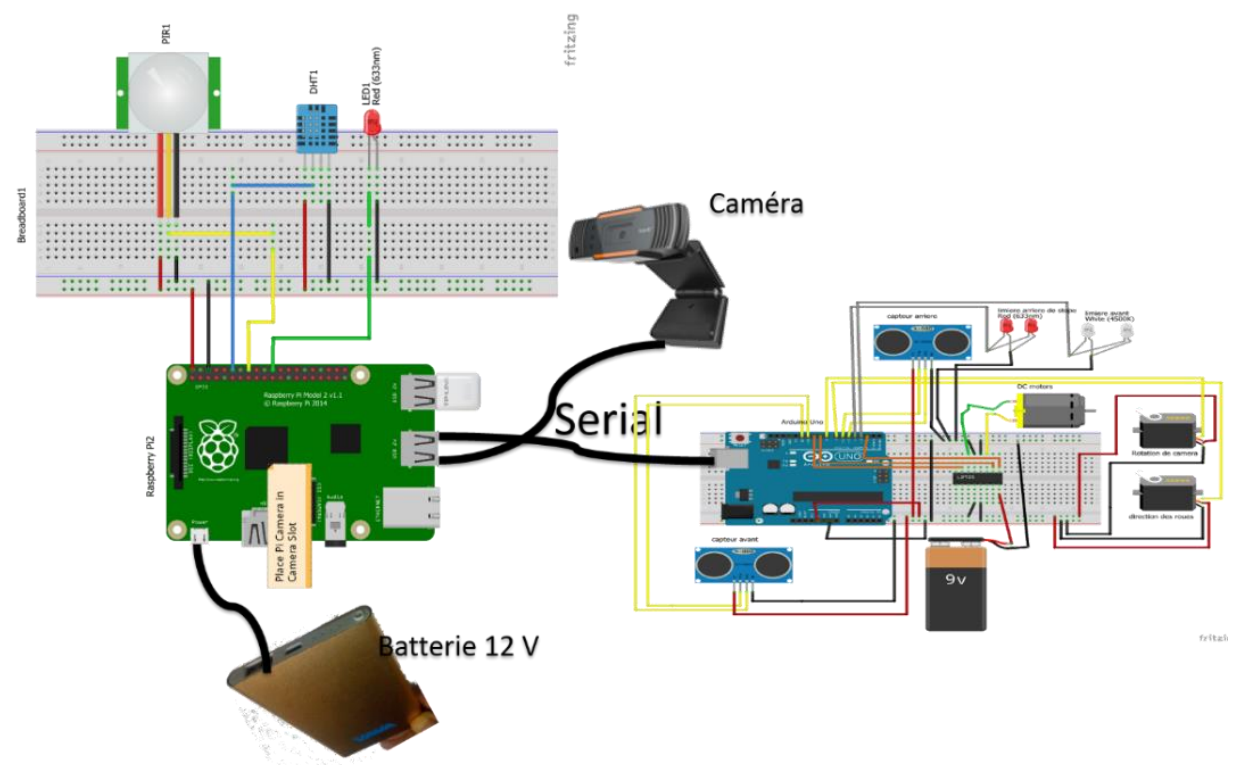

Fig 6. The general electronic Robot circuit

\section{System behavior results}

The following figure shows the final shape of the robot. The robot is equipped with sensors and a surveillance camera.

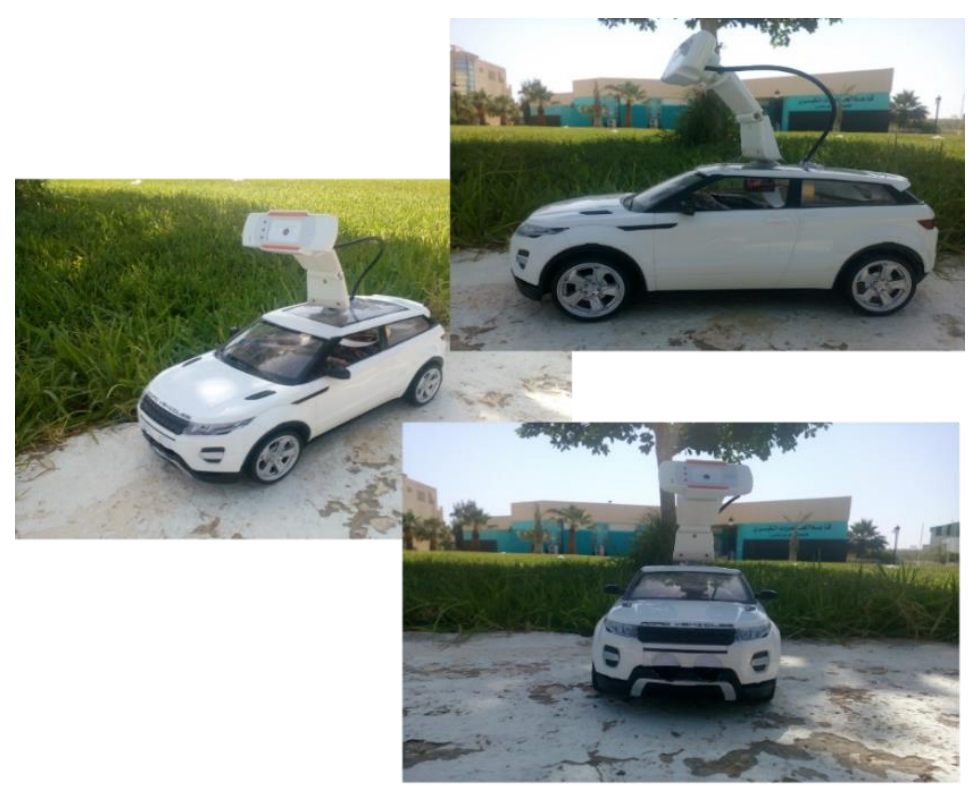

Fig 7. Visualization of the Robot 


\subsection{Robot Mobility}

The robot is moving manually, thanks to the user's commands received by the robot via a smartphone. These commands are sent using the wireless network (WIFI) to the Raspberry PI. The Raspberry PI transmit orders via serial cable to the Arduino board to be executed.

\subsection{Robot sensing}

The Robot sensed the temperature and humidity of the environment and then stored the data in its own database. It also notified the user in the event of significant changes in temperature and humidity.

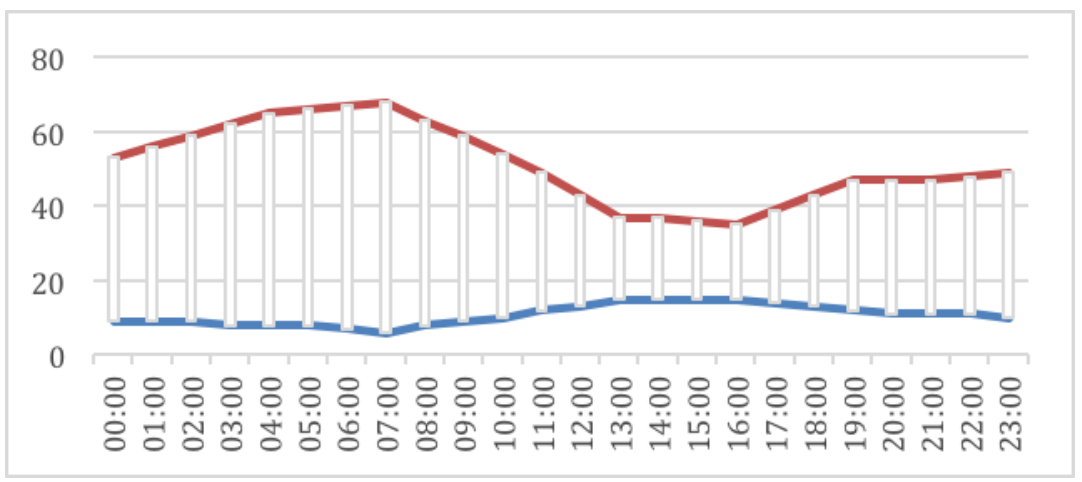

\subsection{Surveillance camera}

We supplied the robot with a steerable camera. It is controlled remotely by sent commands. The camera server (motion) sends the captured images to the user in real time and directly. Motion is a fairly complete surveillance system. It is extremely customizable: motion detection, frame-by-frame recording, video recording, time lapse.

\section{Conclusion}

In this paper we implemented an IoT Robot robot to sensing the environment for smart home, we implemented this approach using a Raspberry with sensors integration and using MQTT protocol for communications.

As close perspectives of this research work we want to integrate this platform in the cloud computing environment for device data storage, transfer and process.

\section{References}

[ALJ ] Al-Joboury I. M. and E. H. Al-Hemiary, "Internet of Things (IoT): Readme," Qalaai Zanist Sci. J., vol. 2, no. 2, pp. 343-358, 2017.

[VER 17] Vermesan O. et al., "Internet of Robotic Things - Converging Sensing/Actuating, Hyperconnectivity, Artificial Intelligence and IoT Platforms," pp. 97-155, 2017.

[NAI 17] Naik N., "Choice of effective messaging protocols for IoT systems: MQTT, CoAP, AMQP and HTTP," 2017 IEEE Int. Symp. Syst. Eng. ISSE 2017 - Proc., 2017.

[WAR 11] J.-D. Warren, J. Adams, and H. Molle, Arduino Robotics. 2011.

[ALS XX] S. H. Alsamhi, O. Ma, and M. S. Ansari, "Artificial Intelligence-Based Techniques for Emerging Robotics Communication : A Survey and Future Perspectives."

[PIY 13] R. Piyare, "Internet of Things : Ubiquitous Home Control and Monitoring System using Android based Smart Phone," Int. J. Internet Things, vol. 2, no. 1, pp. 5-11, 2013.

[MER 16] S. Meraghni, L. S. Terrissa, N. Zerhouni, C. Varnier, and S. Ayad, "A post-prognostics decision framework for cell site using Cloud computing and Internet of Things," 2016 2nd Int. Conf. Cloud Comput. Technol. Appl., no. May 2016, pp. 310-315, 2016. 
[BOZ ] Z. Bozdogan and R. Kara, "Layered model architecture for internet of things," J. Eng. Res. Appl. Sci., vol. 4, no. June, pp. 260-264, 2015.

[CHE 70] Y. Chen and H. Hu, "Internet of intelligent things and robot as a service," Simul. Model. Pract. Theory, vol. 34, pp. 159-171, 1970.

[ZHA 15] C. W. Zhao, J. Jegatheesan, and S. C. Loon, "Exploring IOT Application Using Raspberry Pi," Int. J. Comput. Networks Appl. Vol., vol. 2, no. 1, pp. 27-34, 2015.

[MAK 14] M. Maksimović, V. Vujović, N. Davidović, V. Milošević, and B. Perišić, "Raspberry Pi as Internet of Things hardware : Performances and Constraints," Des. Issues, vol. 3, no. JUNE, p. 8, 2014.

[YAN 15] W. Yang, S. Qiao, Q. Song, Z. Liu, and J. Yang, "The Design and Implementation of Wireless Temperature and Humidity Control System Based on nRF905," pp. 753-756, 2015.

[SAL 18a] I. SAleh «Internet des Objets (IdO) : Concepts, Enjeux, Défis et Perspectives », Revue Revue : Internet des objets, Numéro 1, Volume 2, 2018, DOI : 10.21494/ISTE.OP.2018.0229, ISSN : 2514-8273.

[SAL 18b] I SAleh, M. Ammi \& S. Szoniecky «Challenges of the Internet of Things: Technology, Use, Ethics », Copublisher ISTE \& Wiley Editions, Londres, 2018, ISBN : 9781786303615. 